\title{
Constructions of copulas with given diagonal (and opposite diagonal) sections and some generalizations
}

https://doi.org/10.1515/demo-2018-0009

Received March 25, 2018; accepted May 26, 2018

\begin{abstract}
We review various methods for constructing bivariate copulas with given diagonal sections from seminal work to the most recent research on copulas with given diagonal and opposite diagonal sections. A survey on a generalization of copulas with given diagonal plane sections in higher dimensions and other sections that generalize the diagonal and opposite diagonal sections is of particular interest.
\end{abstract}

Keywords: Copula, diagonal section, track section

MSC: Primary 60E05, secondary 62E10

Dedicated to Prof. Carlo Sempi on the occasion of his seventieth birthday.

\section{Introduction}

In statistics, copulas are used in the construction of probability distribution functions with fixed margins $[28,55,71]$, and, for this reason, they are extensively used in modeling uncertainty of different types, from probabilistic methods to imprecise probabilities and decision theory (see, e.g. [7, 51]). One of the problems initially studied in copula theory was the construction of copulas given some partial information, for instance, copulas with given sections, and, in particular, with given diagonal sections. The diagonal section of a copula can be used to study the tail dependence of a continuous pair of random variables. Tail dependence has been shown to be useful to describe the dependence between risks in financial and actuarial risk management (see, e.g. [38]). Since then, an ever growing variety of problems has been studied related to that: Copulas with diagonal and opposite diagonal sections and their compatibility, extensions, multidimensional generalizations, etc.

The aim of this paper is to provide a survey of the main results related to the constructions of copulas with given diagonal (and opposite diagonal sections), as well as some of their generalizations in the bivariate case and in higher dimensions.

\section{Preliminaries}

Let $n$ be a natural number such that $n \geq 2$. A ( $n$-dimensional) copula (briefly, $n$-copula) is the restriction to $[0,1]^{n}\left(=\mathbb{I}^{n}\right)$ of a continuous $n$-dimensional distribution function whose univariate margins are uniform on II. Equivalently, an $n$-copula $C$ is a function from $\mathbb{I}^{n}$ to $\mathbb{I}$ satisfying the following conditions:

Juan Fernández-Sánchez: Research Group of Mathematical Analysis, University of Almería, 04120 Almería, Spain, E-mail: juanfernandez@ual.es

^Corresponding Author: Manuel Úbeda-Flores: Department of Mathematics, University of Almería, 04120 Almería, Spain, E-mail: mubeda@ual.es 
(C1) For every $\mathbf{u}=\left(u_{1}, \ldots, u_{n}\right)$ in $\mathbb{I}^{n}, C(\mathbf{u})=0$ if at least one coordinate of $\mathbf{u}$ is 0 , and $C(\mathbf{u})=u_{k}$ whenever all coordinates of $\mathbf{u}$ are 1 except, maybe, $u_{k}$; and

(C2) the $n$-increasing property: for every $\mathbf{a}=\left(a_{1}, \ldots, a_{n}\right)$ and $\mathbf{b}=\left(b_{1}, \ldots, b_{n}\right)$ in $\mathbb{I}^{n}$ such that $a_{k} \leq b_{k}$ for all $k=1,2, \ldots, n, V_{C}([\mathbf{a}, \mathbf{b}]):=\sum \operatorname{sgn}(\mathbf{c}) \cdot C(\mathbf{c}) \geq 0$, where the sum is taken over all the vertices $\mathbf{c}=\left(c_{1}, \ldots, c_{n}\right)$ of $[\mathbf{a}, \mathbf{b}]:=x_{i=1}^{n}\left[a_{i}, b_{i}\right]$ (i.e., each $c_{k}$ is equal to either $a_{k}$ or $\left.b_{k}\right)$, and $\operatorname{sgn}(\mathbf{c})=1$ if $c_{k}=a_{k}$ for an even number of $k$ 's, and $\operatorname{sgn}(\mathbf{c})=-1$ otherwise.

The importance of copulas in statistics is described in the following result due to A. Sklar [87]: Let $X_{1}, \ldots, X_{n}$ be $n$ random variables with joint distribution function $H$ and one-dimensional marginal distributions $F_{1}, \ldots, F_{n}$, respectively. Then there exists an $n$-copula $C$ (which is uniquely determined on $\times_{i=1}^{n} \operatorname{Range} F_{i}$ ) such that $H(\mathbf{x})=C\left(F_{1}\left(x_{1}\right), \ldots, F_{n}\left(x_{n}\right)\right)$ for all $\mathbf{x}=\left(x_{1}, \ldots, x_{n}\right) \in[-\infty, \infty]^{n}$. If $F_{1}, \ldots, F_{n}$ are continuous, then $C$ is unique. For a complete and constructive proof of Sklar's theorem, see, e.g. [13, 93]; and for a complete survey on copulas, see $[28,71]$.

Let $\mathcal{C}_{n}$ denote the set of all $n$-copulas, and let $\Pi_{n}$ denote the copula of independent random variableshere the subscript denotes dimension-, i.e., $\Pi_{n}(\mathbf{u})=\prod_{i=1}^{n} u_{i}$ for every $\mathbf{u}$ in $\mathbb{I}^{n}$.

Any $n$-copula $C$ induces on the class of Borel sets of $\mathbb{I}^{n}$, denoted by $\mathcal{B}\left(\mathbb{I}^{n}\right)$, an $n$-stochastic measure $\mu_{C}$ satisfying $\mu_{C}([\mathbf{a}, \mathbf{b}]):=V_{C}([\mathbf{a}, \mathbf{b}])$. The support of an $n$-copula $C$ is the complement of the union of all open subsets of $\mathbb{I}^{n}$ with $\mu_{C}$-measure zero. By the Lebesgue decomposition theorem (see, e.g. [6]), we have $\mu_{C}=$ $\mu_{C}^{a c}+\mu_{C}^{s}$, where $\mu_{C}^{a c}$ is a measure on $\mathcal{B}\left(\mathbb{I}^{n}\right)$ that is absolutely continuous with respect to the $n$-dimensional Lebesgue measure, and $\mu_{C}^{s}$ is a measure on $\mathcal{B}\left(\mathbb{I}^{n}\right)$ that is singular with respect to the Lebesgue measure on $\mathbb{I}^{n}$. Therefore, for all $\mathbf{u} \in \mathbb{I}^{n}$, we have $C(\mathbf{u})=C_{a c}(\mathbf{u})+C_{s}(\mathbf{u})$, where $C_{a c}(\mathbf{u})=\mu_{C}^{a c}([\mathbf{0}, \mathbf{u}])$ and $C_{s}(\mathbf{u})=\mu_{C}^{s}([\mathbf{0}, \mathbf{u}])$ are the absolutely continuous and singular component of an $n$-copula $C$, respectively. Thus, $C$ is said to be absolutely continuous (respectively, singular) when $C_{s} \equiv 0$ (respectively, $C_{a c} \equiv 0$ ).

A 2-copula $C$ is called symmetric if $C(u, v)=C(v, u)$ for every $(u, v)$ in $\mathbb{I}^{2}$.

The concept of a quasi-copula is a more general notion than that of a copula. It was introduced in [2] (for the bivariate case) and [78] (for the multivariate case) in order to show that certain operations on univariate distributions are derivable from operations on random variables defined on the same probability space. An $n$-dimensional quasi-copula (briefly $n$-quasi-copula) is a function $Q$ from $\mathbb{I}^{n}$ to $\mathbb{I}$ satisfying condition (C1) for $n$-copulas and, in place of $(\mathrm{C} 2)$, the weaker conditions:

(Q1) $Q$ is non-decreasing in each variable; and

(Q2) the 1-Lipschitz condition $|Q(\mathbf{u})-Q(\mathbf{v})| \leq \sum_{i=1}^{n}\left|u_{i}-v_{i}\right|$ for all $\mathbf{u}, \mathbf{v} \in \mathbb{I}^{n}$

(see [50] for the case $n=2$, and [12] for $n>2$ ). Let $Q_{n}$ denote the set of all $n$-quasi-copulas.

Every $n$-quasi-copula $Q$ satisfies the following inequalities $W_{n}(\mathbf{u})=\max \left(\sum_{i=1}^{n} u_{i}-n+1,0\right) \leq C(\mathbf{u}) \leq$ $\min \left(u_{1}, \ldots, u_{n}\right)=M_{n}(\mathbf{u}) . M_{n}$ is an $n$-copula for all $n \geq 2$, and $W_{n}$ is a proper $n$-quasi-copula, i.e. a $n$-quasicopula but not an $n$-copula (except if $n=2$, which is a 2-copula). These bounds are known as the FréchetHoeffding bounds. For illustrating the differences between copulas and quasi-copulas-in the bivariate case or in higher dimensions-, we refer to [4, 19, 45, 70, 74, 77], and for a complete survey on quasi-copulas, see [84].

We will use some additional notation. Given two real numbers $a$ and $b$, we denote $a \vee b=\max (a, b)$ and $a \wedge b=\min (a, b)$.

\section{Construction of copulas with given diagonal sections}

In this section we first recall the concept of diagonal section of an $n$-copula, then, we recall the most salient constructions of copulas with given diagonal sections and some generalizations, both in the bivariate case and in higher dimensions. 


\subsection{The diagonal section of an $n$-copula}

The diagonal section of an $n$-copula $C$ is the function $\delta_{C}: \mathbb{I} \longrightarrow \mathbb{I}$ defined by $\delta_{C}(t)=C(t, \ldots, t)$. The diagonal section $\delta_{C}$ of an $n$-copula $C$ satisfies the following conditions:

(D1) $\delta_{C}(1)=1$,

(D2) $\delta_{C}(t) \leq t$ for all $t \in \mathbb{I}$,

(D3) $0 \leq \delta_{C}\left(t^{\prime}\right)-\delta_{C}(t) \leq n\left(t^{\prime}-t\right)$ for all $t, t^{\prime} \in \mathbb{I}$ such that $t \leq t^{\prime}$.

The diagonal section of an $n$-copula has several probabilistic interpretations. For instance, if $U_{1}, \ldots, U_{n}$ are $n$ continuous random variables defined on the same probability space with associated $n$-copula $C$, then $\delta_{C}$ is the restriction to $\mathbb{I}$ of the distribution function of $\max \left(U_{1}, \ldots, U_{n}\right)$. Furthermore, for $n=2, \delta_{C}$ can be used to study the tail dependence of a continuous pair of random variables $(X, Y)$ with respective marginal distribution functions $F$ and $G[55,71]$ : The upper and lower tail dependence parameters $\lambda_{U}$ and $\lambda_{L}$, which are defined as $\lambda_{U}=\lim _{t \rightarrow 1^{-}} \operatorname{Pr}\left[Y>G^{(-1)}(t) \mid X>F^{(-1)}(t)\right]$ and $\lambda_{L}=\lim _{t \rightarrow 0^{+}} \operatorname{Pr}\left[Y \leq G^{(-1)}(t) \mid X \leq F^{(-1)}(t)\right]$ (if the limits exist), respectively, and where $A^{(-1)}$ denotes the quasi-inverse of $A$, i.e. $A^{(-1)}(t)=\inf \{x \in \mathbb{R}: A(x) \geq t\}$, can be computed as follows: $\lambda_{U}=2-\delta_{C}^{\prime}\left(1^{-}\right)$and $\lambda_{L}=\delta_{C}^{\prime}\left(0^{+}\right)$. Copulas are used to build models for dependence between risks in financial and actuarial risk management, and tail dependence has been shown to be useful to describe this dependence (see, e.g. [23, 38, 62]). For the case of higher dimensions, which is more complicated, we refer to [22, 46, 61].

\subsection{First constructions of copulas with given diagonal sections}

Any function $\delta: \mathbb{I} \longrightarrow \mathbb{I}$ that satisfies (D1)-(D3) is called an $n$-dimensional diagonal function (or $n$-diagonal; or simply diagonal, if there is no confusion). The set of all ( $n$-dimensional) diagonals is denoted by $\mathcal{D}_{n}$.

It is clear that the diagonal section of an $n$-copula is a diagonal. Conversely, for any $n$-diagonal $\delta$, does there exist at least one $n$-copula with diagonal section $\delta$ ? The answer is "yes", and was first given in [48] for the bivariate case, and in [12] for the general case (see also [53, 54, 68]). So we have

Theorem 1 ([53]). If $\delta \in \mathcal{D}_{n}$, then there exists an n-copula $C$ such that $\delta=\delta_{C}$.

Proof. Let $\delta \in \mathcal{D}_{n}$, and consider the function $C_{n, \delta}: \mathbb{I}^{n} \longrightarrow \mathbb{I}$ defined by

$$
C_{n, \delta}(\mathbf{u})=\frac{1}{n} \sum_{i=1}^{n} \min \left\{f\left(u_{\tau^{i}(1)}\right), \ldots, f\left(u_{\tau^{i}(n-1)}\right), \delta\left(u_{\tau^{i}(n)}\right)\right\},
$$

where $f: \mathbb{I} \longrightarrow \mathbb{I}$ is the function given by

$$
f(t)=\frac{n t-\delta(t)}{n-1}
$$

and $\tau^{i}(k)=k+i \bmod n$.

Using conditions (D1)-(D3) we have $f(0)=0, f(1)=1$ and $f$ is continuous and non-decreasing, and hence it is a distribution function. Thus, each summand

$$
\min \left\{f\left(u_{\tau^{i}(1)}\right), \ldots, f\left(u_{\tau^{i}(n-1)}\right), \delta\left(u_{\tau^{i}(n)}\right)\right\}
$$

is a distribution function as well, whence $C_{n, \delta}$ is non-decreasing. Moreover, $f(0)=\delta(0)=0$ implies $C_{n, \delta}\left(u_{1}, \ldots, u_{i-1}, 0, u_{i+1}, \ldots, u_{n}\right)=0$, and since $f(1)=\delta(1)=1$, we have

$$
C_{n, \delta}(1, \ldots, 1, t, 1, \ldots, 1)=\frac{1}{n}(\delta(t)+(n-1) f(t))=\frac{1}{n}\left(\delta(t)+(n-1) \frac{n t-\delta(t)}{n-1}\right)=t .
$$

Finally, since $\delta(t) \leq t \leq f(t)$ for every $t \in \mathbb{I}$, we have

$$
C_{n, \delta}(\mathbf{t})=\frac{1}{n} \sum_{i=1}^{n}(f(t) \wedge \delta(t))=\delta(t)
$$


Therefore, $C_{n, \delta}$ is an $n$-copula with diagonal section $\delta$.

Example 1. If $\delta(t)=\delta_{W_{n}}(t)=(n t-n+1) \vee 0$ for all $t$ in $\mathbb{I}$, then the $n$-copula $C_{n, \delta}$ defined in (1) has a mass $\frac{1}{n}$ uniformly distributed on each of the segments with extremes $\frac{n-1}{n} \mathbf{e}_{i}$ and $\frac{n-1}{n} \mathbf{1}+\frac{1}{n} \mathbf{e}_{i}$, where $\mathbf{e}_{i}$ is the vector whose all components are null except the $i$-th coordinate, which is 1 , and $\sum_{i=1}^{n} \mathbf{e}_{i}=\mathbf{1}$. In Figure 1, the supports of the copulas $C_{2, \delta}$ and $C_{3, \delta}$ and the bivariate margins (which are the same) of $C_{3, \delta}$ are given.
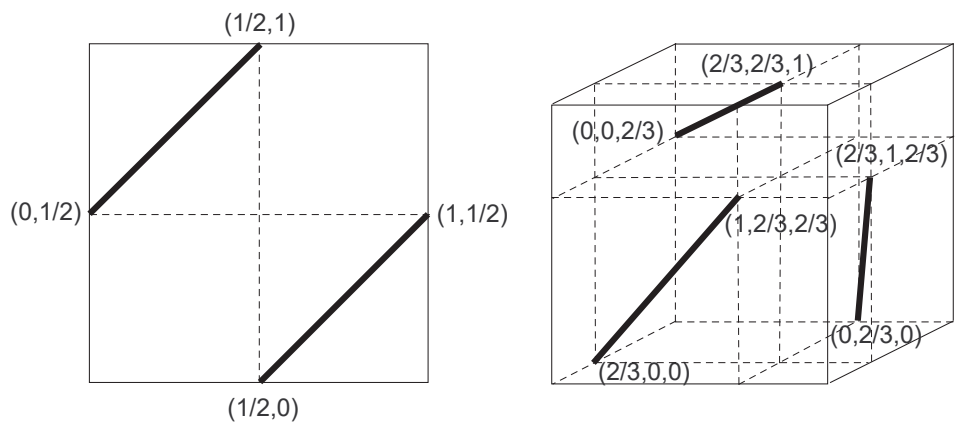

(a) Copulas $C_{2, \delta}$ (left) and $C_{3, \delta}$ (right)

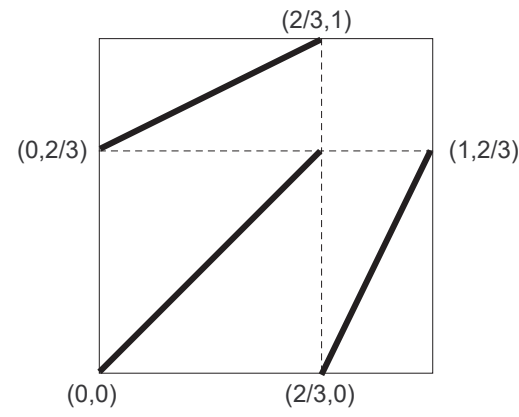

(b) Bivariate margins of $C_{3, \delta}$

Figure 1: In (a) the supports of the copulas $C_{2, \delta}$ and $C_{3, \delta}$, and in (b) the support of the bivariate margins of $C_{3, \delta}$ of Example 1.

Let $\mathcal{C}_{n, \delta}$ denote the set of all $n$-copulas $C$ whose diagonal section $\delta_{C}$ is $\delta$. The definition of diagonal section of an $n$-quasi-copula is the same to that for an $n$-copula, and $Q_{n, \delta}$ will denote the set of all $n$-quasi-copulas $Q$ whose diagonal section $\delta_{Q}$ is $\delta$. We note $\mathcal{C}_{n, \delta} \subseteq Q_{n, \delta}$.

If $\delta \in \mathcal{D}_{n}$, then $\mathcal{C}_{n, \delta}$ is a singleton set if, and only if, $\delta(t)=t$ for all $t$ in $\mathbb{I}$ (see [26] for details).

If $n=2$ in (1), we have the first example of a 2-copula in $\mathcal{C}_{2, \delta}$, and which is given by

$$
C_{\delta}^{\mathrm{FN}}(u, v)=\min \left\{u, v, \frac{\delta(u)+\delta(v)}{2}\right\}, \quad(u, v) \in \mathbb{I}^{2} .
$$

(see [48, 72]). Copulas given by (2) are called diagonal copulas, and they are (symmetric and) singular. We note that $C_{\delta}^{\mathrm{FN}}$ is a hairpin 2-copula if, and only if, $\delta$ is a strict diagonal, i.e. $\delta(t)<t$ for all $t$ in ]0, 1[ (see $[30,72,82,86]$ for details).

A second example (again, in the bivariate case) was given in [49] with the Bertino copulas (based on the seminal paper [8]). This family of 2-copulas, given by

$$
C_{\delta}^{\mathrm{Ber}}(u, v)=u \wedge v-\min \{t-\delta(t): t \in[u \wedge v, u \vee v]\},
$$


are (symmetric and) singular as well. We note that the Bertino 2-copula is a result of a Lipschitz interpolation [7] (see also [5, 59]).

For the diagonal $\delta(t)=t^{2}$, the copulas $C_{\delta}^{\mathrm{FN}}$ y $C_{\delta}^{\mathrm{Ber}}$ (with their corresponding contours) are provided in Figure 2.

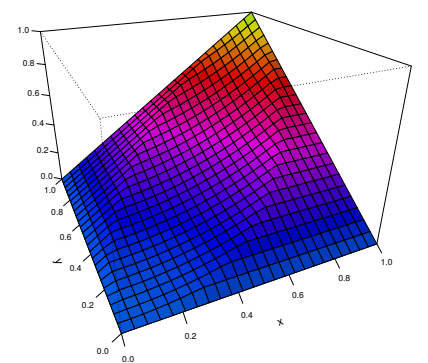

(a) Diagonal

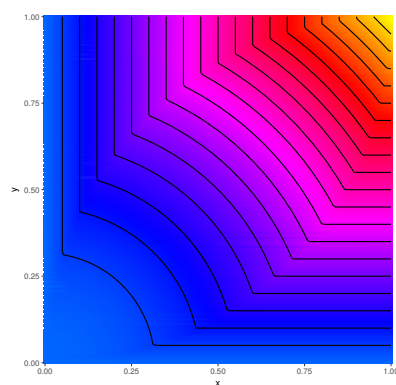

(c) Diagonal

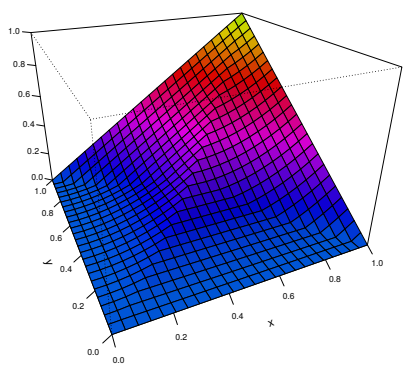

(b) Bertino

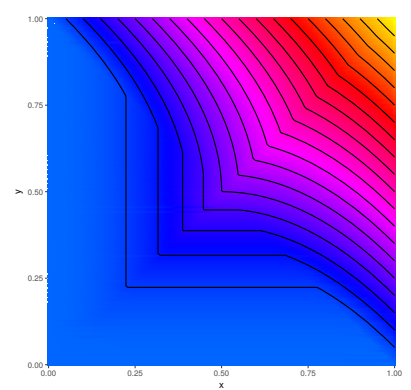

(d) Bertino

Figure 2: In the first row, the 2-copulas $C_{\delta}^{\mathrm{FN}}$ y $C_{\delta}^{\mathrm{Ber}}$ with diagonal $\delta(t)=t^{2}$, and in the second row their corresponding contours.

It is possible to construct a multivariate version of Bertino copulas. Let $\delta \in \mathcal{D}_{n}$, and let $C_{n, \delta}^{\text {Ber }}$ be the function-in fact, the $n$-quasi-copula-defined by

$$
C_{n, \delta}^{\mathrm{Ber}}(\mathbf{u})=\sup \left\{\delta(t)-\sum_{i=1}^{n}\left(\left(t-u_{i}\right) \vee 0\right): t \in\left[u_{(1)}, u_{(n)}\right]\right\},
$$

where $u_{(j)}$ is the $j$-th component of $\mathbf{u} \in \mathbb{I}^{n}$ rearranged in increasing order. If $\delta$ is $n /(n-1)$-Lipschitz continuous, i.e. $\left|\delta(t)-\delta\left(t^{\prime}\right)\right| \leq(n /(n-1))\left|t-t^{\prime}\right|$ for all $t, t^{\prime}$ in $\mathbb{I}$, then $C_{n, \delta}^{\text {Ber }}$ is an $n$-copula (see [3] for details).

Open Problem 1 ([67]). Characterize all the diagonals $\delta \in \mathcal{D}_{n}$ such that $C_{n, \delta}^{\mathrm{Ber}} \in \mathcal{C}_{n}^{\delta}$.

Another class of 2-copulas $C$ with given diagonal section can be derived by means of the functional equation $C(u, v)+|u-v|=C(u \vee v, u \vee v)$, whenever $C(u, v)>0$. It is studied in [31], and is based on the seminal work [63]. These 2-copulas, denoted by $M_{\delta}$, are given by

$$
M_{\delta}(u, v)=(\delta(u \vee v)-|u-v|) \vee 0,
$$

provided that the diagonal $\delta \in \mathcal{D}_{2}$ satisfies $\delta(a)=0$ for some $a \in[0,1 / 2]$ and the function $\delta(t)-t$ is nondecreasing on $[a, 1]$. Note that every 2-copula $M_{\delta}$ is a Bertino copula. 


\subsection{Copula diagonal splice}

All the families of 2-copulas seen until now are symmetric, and this is something that limits their use in many applications. A method for constructing (symmetric and) non-symmetric 2-copulas-called diagonal patchwork copula in [35] and copula diagonal splice in [76] (see also [32])-consists of creating new functions by joining portions of two 2-(quasi-)copulas with a common diagonal section. Let $\delta \in \mathcal{D}_{2}$, and let $Q^{1}$ and $Q^{2}$ be two 2-quasi-copulas in $Q_{2, \delta}$. The diagonal splice (or diagonal patchwork) of $Q^{1}$ and $Q^{2}$ is the function $Q^{1} / Q^{2}$ defined by

$$
\left(Q^{1} / Q^{2}\right)(u, v):= \begin{cases}Q^{1}(u, v), & \text { if } u \leq v, \\ Q^{2}(u, v), & \text { if } u>v .\end{cases}
$$

For $\delta \in \mathcal{D}_{2}$, the diagonal splice of two 2-quasi-copulas is a 2-quasi-copula in $Q_{2, \delta}$ (see [76]).

If $C^{1}$ and $C^{2}$ are 2-copulas with a common diagonal section $\delta$, then the diagonal splice $C^{1} / C^{2}$ can be a proper quasi-copula or a copula. The following result summarizes necessary and sufficient conditions for the diagonal splice of two copulas to be a copula (see $[35,76]$ for more details).

Proposition 2. Let $\delta \in \mathcal{D}_{2}$, and let $C^{1}$ and $C^{2}$ be two 2-copulas in $\mathrm{C}_{2, \delta}$.

(i) The diagonal splice $C^{1} / C^{2}$ is a copula in $C_{2, \delta}$ if, and only, if $C^{1}(u, v)+C^{2}(v, u) \leq \delta(u)+\delta(v)$ for every $(u, v) \in \mathbb{I}^{2}$ such that $u \leq v$.

(ii) If $\max \left(C^{1}(u, v), C^{2}(u, v)\right) \leq C_{\delta}^{\mathrm{FN}}(u, v)$ for all $(u, v)$ in $\mathbb{I}^{2}$, then the diagonal splice $C^{1} / C^{2}$ is a copula in $\mathcal{C}_{2, \delta}$.

(iii) If $C^{1}$ and $C^{2}$ are symmetric, then the diagonal splice $C^{1} / C^{2}$ is a copula in $\mathrm{C}_{2, \delta}$.

Figure 3 shows that we can construct a copula from the diagonal splice of two proper quasi-copulas (see [76] for details).
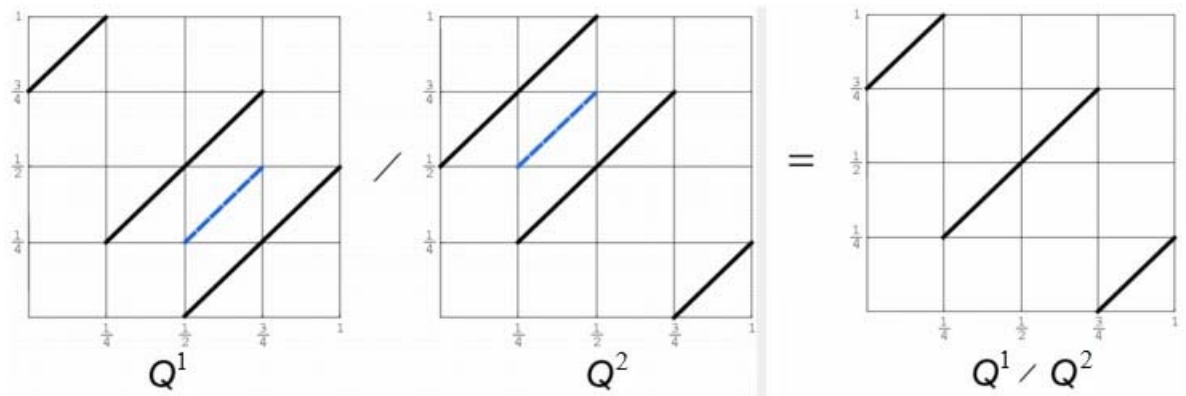

Figure 3: Example for which the diagonal splice of two proper-quasi-copulas is a copula.

\subsection{A construction that generalizes the family $C_{\delta}^{\mathrm{FN}}$}

In this subsection we recall a non-symmetric family of 2-copulas that generalizes the symmetric family $C_{\delta}^{\mathrm{FN}}$ for $\delta \in \mathcal{D}_{2}$ given by (2). Let $I(\delta)=\{t \in] 0,1\left[: \exists \delta^{\prime}(t)\right\}$, and let $\mathcal{D}_{0}$ be the subset of the diagonals $\delta \in \mathcal{D}_{2}$ for which $\delta(t)<t$ for all $t \in] 0,1\left[\right.$ and such that there exists $\varepsilon_{\delta}>0$ for which it holds $\sup \left\{\delta^{\prime}(t): t \in I(\delta)\right\}=\frac{2}{1+2 \varepsilon_{\delta}}<2$. Then, for every diagonal $\delta \in \mathcal{D}_{0}$ and for all $\alpha \in\left[\frac{1}{2}-\varepsilon_{\delta}, \frac{1}{2}+\varepsilon_{\delta}\right]$, the function $K_{\delta, \alpha}$ defined on $\mathbb{I}^{2}$ by

$$
K_{\delta, \alpha}(u, v)=\min \{u, v, \alpha \delta(u)+(1-\alpha) \delta(v)\}
$$

is a copula with diagonal section $\delta$ (see [35]). 


\subsection{Construction of absolutely continuous copulas}

The construction methods that we have reviewed above provide copulas with given diagonal sections having, in general, a singular component. However, if we have an absolutely continuous copula with given diagonal section $\delta$, it is possible to construct (infinitely many) absolutely continuous copulas with diagonal section $\delta$. In fact, we have the following result.

Proposition 3. A diagonal $\delta \in \mathcal{D}_{n}$ is the diagonal section of an absolutely continuous $n$-copula if, and only if, the set $\{t \in \mathbb{I}: \delta(t)=t\}$ has Lebesgue measure 0 .

The bidimensional case of Proposition 3 is proved in [27], and the general case in [53]. Moreover, for the bidimensional case, we have the following result.

Proposition 4 ([29]). Let $\delta \in \mathcal{D}_{2}$. The following statements are equivalent:

(a) $\delta$ is the diagonal of an absolutely continuous copula with full support;

(b) $\delta(t)<t$ for every $t \in] 0,1\left[\right.$ and there is no interval $J \subseteq \mathbb{I}$ such that either $\delta^{\prime}=0$ on $J$ or $\delta^{\prime}=2$ on $J$.

Different methods for the construction of absolutely continuous copulas with given diagonal sections can be found in [16, 27, 33, 39]. For instance, in [27], if $\delta \in \mathcal{D}_{0}$, the function $C_{\delta}$ defined on $\mathbb{I}^{2}$ by

$$
C_{\delta}(u, v)=\frac{1}{2 \varepsilon_{\delta}} \int_{\frac{1}{2}-\varepsilon_{\delta}}^{\frac{1}{2}+\varepsilon_{\delta}} K_{\delta, \alpha}(u, v) d \alpha,
$$

where $K_{\delta, \alpha}$ is the 2-copula given in (5), is an absolutely continuous 2-copula with diagonal section equal to $\delta$. For constructions of absolutely continuous $n$-copulas with given diagonal sections, see [53].

\subsection{Copula-entropy}

If $C$ is an absolutely continuous $n$-copula with density $c$, we recall that the Shannon-entropy-or copulaentropy-(see the seminal work [85]) is the value given by $-\int_{\mathbb{I}^{n}} c(\mathbf{x}) \ln c(\mathbf{x}) d \mathbf{x}$ (the entropy is $-\infty$ if the copula is not absolutely continuous). In [9], it is shown that there exists an $n$-copula with diagonal section $\delta$ and finite entropy if, and only if, $\delta$ satisfies the following condition: $\int_{\mathbb{I}}|\ln (t-\delta(t))| d t<+\infty$. In this case, there exists a unique $n$-copula with the property that its entropy is equal to the maximum of the entropies of the copulas of the set $e_{n, \delta}$. Furthermore, the density of this copula is described. For example, in the bivariate case, for $\delta(t)=\delta_{\Pi_{2}}(t)=t^{2}$, the copula that maximizes the entropy is $\Pi_{2}$, and for $\delta(t)=\delta_{W_{2}}(t)=0 \vee(2 t-1)$, such copula has zero density in $L=\left[0, \frac{1}{2}\right]^{2} \cup\left[\frac{1}{2}, 1\right]^{2}$ and density 2 in $\mathbb{I}^{2} \backslash L$.

\subsection{Best-possible bounds on sets of 2-copulas with given diagonal section}

Any set of $n$-copulas sharing a particular statistical property (e.g. $n$-copulas with the same diagonal section) is guaranteed to have point-wise best-possible bounds within the set $Q_{n}$ (see [75] for the bivariate case and [80] for the multivariate case). This is due to the fact that $\left(Q_{n}, \leq\right)$ is a complete lattice, i.e. for every subset $S \subseteq Q_{n}, \vee S$ and $\wedge S$ are in $Q_{n}$. We note that $Q_{n}$ is order-isomorphic to the Dedekind-MacNeille completion of the set $\mathcal{C}_{n}$ only if $n=2$ (see [44, 73]). Here we tackle the problem of finding the best-possible bounds on sets of 2-copulas with a given diagonal section.

For any $\delta \in \mathcal{D}_{2}$, consider the function

$$
A_{\delta}(u, v)=\min \left\{u, v,(u \vee v)-h_{\delta}(u, v)\right\}, \quad(u, v) \in \mathbb{I}^{2},
$$

where $h_{\delta}(u, v)=\max \{t-\delta(t): t \in[u \wedge v, u \vee v]\}$, which belongs to $Q_{2, \delta}$ and satisfies $Q \leq A_{\delta}$ for any $Q \in Q_{2, \delta}$ (see [75]). The following result summarizes different ways to construct copulas and quasi-copulas with given 
diagonal sections, and some of their known properties (see [41, 48, 72, 75, 76, 91, 92]), where $\bar{C}_{\delta}$ denotes the best-possible upper bound of the set $\mathcal{C}_{2, \delta}$ and the ordinal sum of copulas can be consulted in [71].

Proposition 5. Let $\delta \in \mathcal{D}_{2}$, and let $C_{\delta}^{\mathrm{FN}}, C_{\delta}^{\mathrm{Ber}}$ and $A_{\delta}$ be the functions given by (2), (3) and (6), respectively. Then the following statements hold:

(i) $A_{\delta}$ is a copula if, and only if, $A_{\delta}=C_{\delta}^{\mathrm{FN}}$. As well, $A_{\delta}$ is a copula if, and only if, it is an ordinal sum of $W_{2}$.

(ii) $C_{\delta}^{\text {Ber }} \leq C_{\delta}^{\mathrm{FN}} \leq A_{\delta}$.

(iii) If $C$ is in $\mathcal{C}_{2, \delta}$ and symmetric, then $C \leq C_{\delta}^{\mathrm{FN}}$.

(iv) The best-possible lower bound for both the sets $\mathrm{C}_{2, \delta}$ and $Q_{2, \delta}$ is $C_{\delta}^{\mathrm{Ber}}$, and the best-possible upper bound for the set $Q_{2, \delta}$ is $A_{\delta}$.

(v) $C_{\delta}^{\mathrm{FN}} \leq \bar{C}_{\delta} \leq A_{\delta}$.

(vi) $\bar{C}_{\delta}$ is a copula if, and only if, $\bar{C}_{\delta}=C_{\delta}^{\mathrm{FN}}$.

(vii)If the function $t-\delta(t)$ is quasi-concave, then $\bar{C}_{\delta}=A_{\delta}$.

Open Problem 2 ([76]). Characterize the diagonals $\delta \in \mathcal{D}_{2}$ such that $\bar{C}_{\delta}$ is a copula, i.e. $\bar{C}_{\delta}=C_{\delta}^{\mathrm{FN}}$.

Additional studies of best-possible bounds on copulas with given diagonal sections-based on the constructions previously seen or other different-can be found in $[49,59,60]$, where their existence and "narrowness" with respect to the Fréchet-Hoeffding bounds are studied.

\subsection{Archimedean copulas}

Archimedean copulas are an important class of multivariate dependence models which are used in many applications, such as finance, insurance, or reliability (see, e.g. $[10,11,52,66])$. Let $\varphi: \mathbb{I} \longrightarrow[0,+\infty]$ be a continuous, decreasing and convex function such that $\varphi(1)=0$, and let $\varphi^{[-1]}$ be the pseudo-inverse of $\varphi$, i.e. $\varphi^{[-1]}(t)=\varphi^{-1}(\varphi(0) \wedge t)$ for all $t \in[0,+\infty]$. Then the function $C_{\varphi}$ defined by

$$
C_{\varphi}(\mathbf{u})=\varphi^{[-1]}\left(\sum_{i=1}^{n} \varphi\left(u_{i}\right)\right), \quad \mathbf{u} \in[0,1]^{n},
$$

is an $n$-copula-called Archimedean copula, and where $\varphi$ is the (additive) generator of $C_{\varphi}$ ( $\varphi$ is a strict generator if it is strictly decreasing on $[0,+\infty]$ )-if, and only if, $\varphi^{[-1]}$ is $n$-monotone on $[0,+\infty]$ (see [65] for more details). A characterization of Archimedean $n$-copulas in terms of diagonal generators is provided in [95].

The representation of the diagonal section of an Archimedean 2-copula with a strict generator is unique $[89,94]$. Furthermore, if $C$ is an Archimedean $n$-copula whose diagonal section $\delta_{C}$ satisfies $\delta_{C}^{\prime}\left(1^{-}\right)=n$, then $C$ is uniquely determined by its diagonal-this is known as Frank's condition: see [47] for the bivariate case, and $[40,83]$ for the multivariate case. The following example shows that if Frank's condition is not satisfied then we can construct different Archimedean 2-copulas with the same diagonal section.

Example 2 ([1]). Let $\varphi$ and $\delta$ the real functions defined by $\varphi(x)=(\ln x)^{2}$ and $\delta(x)=x^{\sqrt{2}}$, respectively. For $\beta \in] 0,1]$, define $t_{\beta}: \mathbb{R}^{+} \longrightarrow \mathbb{R}^{+}$in the following manner: $t_{\beta}(x)=x+2^{n} \beta \sin \left(\frac{x}{2^{n}}\right)$, when $2^{n+1} \pi \leq x \leq 2^{n+2} \pi$ and $n \in \mathbb{Z}$, and $t_{\beta}(0)=0$. Note that $t_{\beta}$ is a continuous and strictly increasing function satisfying $t_{\beta}(2 x)=2 t_{\beta}(x)$. Define $\varphi_{\beta}=t_{\beta} \circ \varphi$. When $\left.\left.\beta \in\right] 0, \frac{1}{1+8 \pi}\right]$, the functions $\varphi_{\beta}$ are convex and $\varphi_{\beta}(1)=0$, so for each $\left.\left.\beta \in\right] 0, \frac{1}{1+8 \pi}\right]$ we have a copula $C_{\beta}$ generated by $\varphi_{\beta}$ whose diagonal section we represent by $\delta_{\beta}$. Since $t_{\beta}(2 x)=2 t_{\beta}(x)$, after some elementary calculations we have $\delta_{\beta}=\delta$. Thus, we obtain $\delta_{\beta_{1}}=\delta_{\beta_{2}}$, whereas $C_{\beta_{1}}=C_{\beta_{2}}$ when $\beta_{1} \neq \beta_{2} \in$ ]0, $\left.\frac{1}{1+8 \pi}\right]$.

Furthermore, if $\varphi$ is the generator of an Archimedean 2-copula, while $\delta_{C}^{\prime}\left(1^{-}\right)<2$ implies $\varphi^{\prime}\left(1^{-}\right)=0$, the converse is not true: For example, this is the case of the generator $\varphi$ defined by $\varphi(t)=-\int_{0}^{1-t} \frac{1}{\ln x} d x$ if $\left.\left.t \in\right] 0,1\right]$, and $\varphi(0)=+\infty$ (see [46] for details). 
Open Problem 3 ([1]). One of the most salient properties of an Archimedean 2-copula $C$ is that it is associative, that is, $C(C(u, v), w)=C(u, C(v, w))$ for all $u, v, w$ in $\mathbb{I}$. The above discussion leads to the following question: Characterize those diagonals that can be extended to associative copulas. May this problem be generalized to higher dimensions? For a study of the associativity of n-copulas (in the sense of Post), see [88]. Based on the fact that a 2-copula is associative if, and only if, it is an ordinal sum of a collection of copulas $\left\{C_{i}\right\}$ such that, for each $i, C_{i}$ is Archimedean [1] (for the definition of ordinal sum in higher dimensions, see [69]), the open problem should be reduced to the case of Archimedean copulas.

Finally, we note that in [24], self-nested diagonals, which are extensions of $k$-fold composition of diagonal sections of an $n$-copula when $k$ belongs to the whole real line, are used to construct Archimedean $n$-copulas.

\subsection{Special types of diagonals}

As it was pointed out above, for $\delta \in \mathcal{D}_{2}$, the functions $C_{\delta}^{\mathrm{FN}}$ and $C_{\delta}^{\mathrm{Ber}}$, given by (2) and (3), respectively, are always copulas. In contrast to these two universal constructions, under other additional assumptions on $\delta$, it is possible to construct new copulas in $\mathrm{C}_{2, \delta}$. This is the case of diagonal functions $\delta$ that simultaneously satisfy $\frac{\delta(t)}{t}$ is non-decreasing and $\frac{\delta(t)}{t^{2}}$ is non-increasing on ]0,1]. Specifically, if $C$ is a 2-copula, the function $L_{\delta}: \mathbb{I}^{2} \longrightarrow \mathbb{I}$ defined by

$$
L_{\delta}(u, v)=\frac{(u \wedge v) \delta_{C}(u \vee v)}{u \vee v},
$$

where the convention $\frac{0}{0}:=0$ is adopted, is a copula whose diagonal section is $\delta$. These copulas were introduced and studied in [37], and are known as lower semilinear copulas. In other words, a copula is lower semilinear if its sections are linear on the segments joining any point of the diagonal of the unit square to the lower side and to the left side of the unit square, respectively. Another type of diagonals which leads to copulas with similar properties to the lower semilinear copulas have been studied in $[18,56]$.

The above properties for $\delta$ allow a particular construction of 2-copulas. However, there is a property for $\delta \in \mathcal{D}_{2}$ which allows different constructions of copulas that have $\delta$ as diagonal: It is the convexity of $\delta$. When $\delta$ is a convex diagonal, the functions $C_{\delta}^{i}$, for $i \in\{1,2,3\}$, are 2-copulas (in the first construction it is necessary that $\delta$ be a homeomorphism):

$$
\begin{aligned}
& C_{\delta}^{1}(u, v)=\delta\left((u \vee v) \delta^{-1}\left(\frac{u \wedge v}{u \vee v}\right)\right), \\
& C_{\delta}^{2}(u, v)=\delta\left(\frac{u \wedge v}{u \wedge v+1-u \vee v}\right)(u \wedge v+1-u \vee v), \\
& C_{\delta}^{3}(u, v)=\min \left\{u, v, \delta\left(\frac{u+v}{2}\right)\right\} .
\end{aligned}
$$

The copulas $C_{\delta}^{1}$ are called quasi-homogeneous copulas. A quasi-homogeneous copula $C$ is characterized by the existence of a continuous, strictly monotonic function $\varphi: \mathbb{I} \longrightarrow \mathbb{R}$ and a function $f: \mathbb{I} \longrightarrow \mathbb{I}$ such that $C(\lambda u, \lambda v)=\varphi^{-1}(f(\lambda) \varphi(C(u, v)))$ for all $u, v, \lambda \in \mathbb{I}$ (see [64]).

The second construction, $C_{\delta}^{2}$, provides a type of copulas known as biconic copulas, and they are those that satisfy that their sections are linear on the segments joining any point of the diagonal of the unit square to the points $(1,0)$ and $(0,1)$. For a complete study, we refer to $[25,42,57]$.

Finally, we note that the family $C_{\delta}^{3}$ was introduced in [26].

For the diagonal $\delta(t)=t^{2}$, the quasi-homogeneous, biconic and $C_{\delta}^{3}$ copulas (with their corresponding contours) are provided in Figure 4.

\subsection{Construction of copulas with given sub-diagonal sections}

The concept of diagonal section was generalized to sub-diagonal section in [79]. Given a 2-copula $C$ and $u_{0} \in$ ]0,1[, the sub-diagonal section $\delta_{u_{0}, C}$ of $C$ at $u_{0}$ is the function $\delta_{u_{0}, C}:\left[0,1-u_{0}\right] \longrightarrow\left[0,1-u_{0}\right]$ defined by $\delta_{u_{0}, C}(t)=\left(u_{0}+t, t\right)$. The sub-diagonal section $\delta_{u_{0}, C}$ of a 2-copula $C$ at $u_{0}$ satisfies the following conditions: 


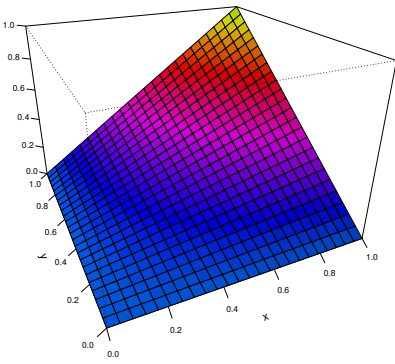

(a) Quasi-homogeneous

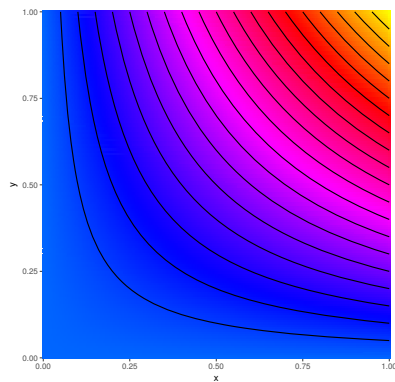

(d) Quasi-homogeneous

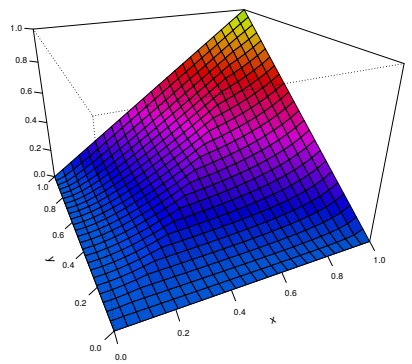

(b) Biconic

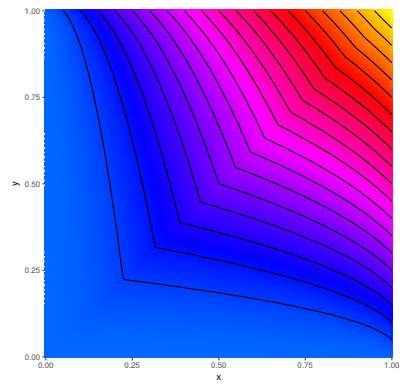

(e) Biconic

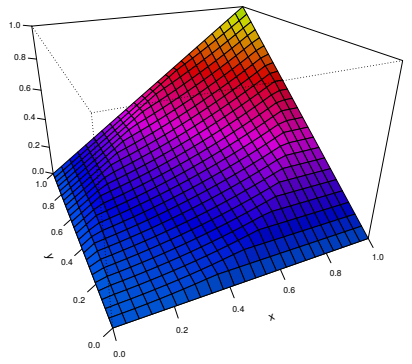

(c) $C_{\delta}^{3}$

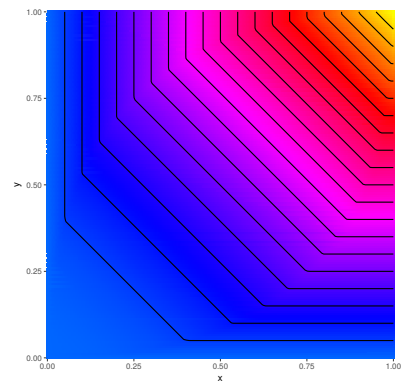

(f) $C_{\delta}^{3}$

Figure 4: In the first row, the quasi-homogeneous, biconic and $C_{\delta}^{3}$ copulas with diagonal $\delta(t)=t^{2}$, and in the second row their corresponding contours.

(SD1) $\delta_{u_{0}, C}\left(1-u_{0}\right)=1-u_{0}$

(SD2) $\delta_{u_{0}, C}(t) \leq t$ for all $t \in\left[0,1-u_{0}\right]$,

(SD3) $0 \leq \delta_{u_{0}, C}\left(t^{\prime}\right)-\delta_{u_{0}, C}(t) \leq 2\left(t^{\prime}-t\right)$ for all $t, t^{\prime} \in\left[0,1-u_{0}\right]$ such that $t \leq t^{\prime}$.

If a 2-copula $C$ is the restriction to the unit square of the distribution function of two random variables $X$ and $Y$ with uniform distribution on $[0,1]$, then $\delta_{u_{0}, C}=\operatorname{Pr}\left[\max \left\{X-u_{0}, Y\right\}<t\right]$.

Given $\left.u_{0} \in\right] 0,1\left[\right.$, any function $\delta_{u_{0}}:\left[0,1-u_{0}\right] \longrightarrow\left[0,1-u_{0}\right]$ that satisfies (SD1)-(SD3) is called a subdiagonal function. The sub-diagonal section of a 2-copula is a sub-diagonal, and conversely, we have the following result, where $\mathcal{C}_{\delta_{u_{0}}}$ denotes the set of all 2-copulas whose sub-diagonal section is $\delta_{u_{0}}$.

Theorem 6 ([79]). For every $\left.u_{0} \in\right] 0,1\left[\right.$ and every sub-diagonal $\delta_{u_{0}}$, there exists a 2-copula $C \in \mathcal{C}_{\delta_{u_{0}}}$.

Furthermore, in [14] the authors show that for every sub-diagonal function there exists an absolutely continuous copula whose diagonal subsection is the given sub-diagonal.

\subsection{Curvilinear sections}

Another generalization of the concept of diagonal section, called curvilinear section, of a 2-copula is provided in [17]. Let $\phi: \mathbb{I} \longrightarrow \mathbb{I}$ be a homeomorphism such that $\phi(0)=0$, and let $G_{\phi}$ be the graph of the function $\phi$. Note that if $\phi(t)=t$ for all $t \in \mathbb{I}$, then $G_{\phi}$ is the diagonal of $\mathbb{I}^{2}$. Let $\mathcal{A}$ denote the set of all homeomorphisms $\phi$ on $\mathbb{I}$. The curvilinear section of a 2-copula $C\left(G_{\phi}\right.$-section of $C$ for short) is the function $G_{C, \phi}: \mathbb{I} \longrightarrow \mathbb{I}$ defined by $G_{C, \phi}(t)=C(t, \phi(t))$. Note that if $\phi(t)=t$ for all $t \in \mathbb{I}$, then $G_{C, \phi}$ is the diagonal section $\delta_{C}$ of $C$. If $\phi \in \mathcal{A}$, the $G_{\phi}$-section of a copula has the following properties:

(i) $0 \vee(t+\phi(t)-1) \leq g(t) \leq t \wedge \phi(t)$ for any $t \in \mathbb{I}$, and

(ii) $0 \leq g\left(t^{\prime}\right)-g(t) \leq t^{\prime}-t+\phi\left(t^{\prime}\right)-\phi(t)$ for any $t \leq t^{\prime} \in \mathbb{I}$. 
Given $G_{\phi} \in \mathcal{A}$, any function $G: \mathbb{I} \longrightarrow \mathbb{I}$ that has these last properties is called a $G_{\phi}$-function. The set of all $G_{\phi^{-}}$ functions is denoted by $S_{\phi}$. The following proposition provides a characterization of the curvilinear section of a copula.

Proposition 7. Let $\phi \in \mathcal{A}$. A function $G: \mathbb{I} \longrightarrow \mathbb{I}$ is a $G_{\phi}$-section of a copula if, and only if, $G \in \mathcal{S}_{\phi}$.

The proof of Proposition 7 uses the fact that, for an increasing compact set $S$ and a 2-copula $C$ the function $T_{S, C}: \mathbb{I}^{2} \longrightarrow \mathbb{I}$ defined by

$$
T_{S, C}(u, v)=\max \left(0, u+v-1, \max _{(a, b) \in S}(C(a, b)-((a-u) \vee 0)-((b-v) \vee 0))\right)
$$

is a copula (see [90]), to prove that

$$
C_{G}(u, v)= \begin{cases}v-\min \left\{\phi(z)-G(z) \mid z \in\left[\phi^{-1}(v), u\right]\right\}, & \text { if } v \leq \phi(u), \\ u-\min \left\{z-G(z) \mid z \in\left[u, \phi^{-1}(v)\right]\right\}, & \text { otherwise }\end{cases}
$$

is the smallest copula with $G_{\phi}$-section $G$. Note that if $\phi(t)=t$ for all $t \in \mathbb{I}$, then $C_{G}$ is the Bertino copula.

\subsection{A trivariate generalization of diagonal section: Diagonal plane sections}

As we have seen, the diagonal section contains the information about the values that an $n$-copula assumes on a set of (Hausdorff) dimension 1. We now provide a possible generalization of this concept when the values of the copula are known on a set of dimension 2. Specifically, we study 3-copulas with known information in a plane (we refer to [34] for further details).

Definition 1. The (1, 2)-diagonal plane section of a 3-copula $C$ is the function $\Delta_{C}^{1,2}: \mathbb{I}^{2} \longrightarrow \mathbb{I}$ given by

$$
\Delta_{C}^{1,2}(u, v)=C(u, u, v)
$$

for every $(u, v) \in \mathbb{I}^{2}$. Similarly, the $(1,3)$-diagonal plane section and the $(2,3)$-diagonal plane section of $C$ are defined by

$$
\Delta_{C}^{1,3}(u, v)=C(u, v, u) \quad \text { and } \quad \Delta_{C}^{2,3}(u, v)=C(v, u, u) \text {, }
$$

respectively.

If $(U, V, W)$ is a random vector (on a suitable probability space) with associated 3-copula $C$, then $\Delta_{C}^{1,2}$ represents the distribution function of $(\max (U, V), W)$ (and similarly for the other diagonal plane sections). Any diagonal plane section is a 2-increasing function and contains the information about the diagonal section of C.

In the sequel, we concentrate on the $(1,2)$-diagonal plane section of the copula $C$ (the other cases can be obtained by a permutation of the arguments of $C$ ), and, for the sake of simplicity, we write $\Delta_{C}:=\Delta_{C}^{1,2}$. The following properties are satisfied:

(PD1) $\Delta_{C}(u, 1)$ is a bidimensional diagonal function;

(PD2) $\Delta_{C}(1, v)=v$ for every $y \in \mathbb{I}$;

(PD3) $\Delta_{C}$ is 2-increasing.

We call a function $\Delta: \mathbb{I}^{2} \longrightarrow \mathbb{I}$ that satisfies properties (PD1)-(PD3) a diagonal plane function.

The following theorem summarizes the most salient results concerning the existence and uniqueness of a 3-copula with a given diagonal plane section.

Theorem 8. Let $\Delta$ be a diagonal plane function. Then:

(i) There exists a 3-copula $C$ whose diagonal plane section is $\Delta$. 
(ii) There exists a unique 3-copula $C$ whose diagonal plane section is $\Delta$ if, and only if, $\Delta(u, 1)=u$ for every $x \in \mathbb{I}$.

(iii) There exists a singular 3-copula $C$ whose diagonal plane section is $\Delta$.

(iv) There exists an absolutely continuous 3-copula $C$ whose diagonal plane section is $\Delta$ if, and only if, $\Delta$ is absolutely continuous and the set $J=\{t \in \mathbb{I}: \Delta(t, 1) \neq t\}$ has Lebesgue measure 1.

Let $\mu_{\Delta}$ be the probability measure associated with a diagonal plane function $\Delta$, and let $\delta(u):=\Delta(u, 1)$ for every $u \in \mathbb{I}$. Then, there exists a family of measures $\left\{\mu_{\Delta, u}\right\}_{u \in \mathbb{I}}$ in the Borel sets of $\mathbb{I}$ such that

$$
\int_{\mathbb{I}^{2}} f(u, v) d \mu_{\Delta}(u, v)=\int_{\mathbb{I}}\left(\int_{\mathbb{I}} f(u, v) d \mu_{\Delta, u}(v)\right) \delta^{\prime}(u) d u
$$

for every Borel map $f: \mathbb{I}^{2} \longrightarrow[0,+\infty]$. Now, let $C_{\delta}$ be a 2-copula with diagonal section $\delta$. Then the function $C_{\Delta}$ given by

$$
C_{\Delta}(u, v, w)=\int_{0}^{u} \int_{0}^{v} \mu_{\Delta, \max (s, t)}([0, w]) d \mu_{C_{\delta}}(s, t) .
$$

is a (singular) 3-copula with diagonal plane function $\Delta$. Moreover, if $\Delta$ is absolutely continuous and the set $J$ has Lebesgue measure 1 then $C_{\Delta}$ is absolutely continuous.

Open Problem 4 ([34]). If we consider $\left(i_{1}, \ldots, i_{k}\right)$ a sub-vector of indices of $\{1,2, \ldots, n\}, i_{1}<i_{2}<\cdots<i_{k}$, we can define the $\left(i_{1}, \ldots, i_{k}\right)$-diagonal hyper-plane section of $C$ as the function $\Delta_{C}^{i_{1}, \ldots, i_{k}}: \mathbb{I}^{n-k+1} \rightarrow \mathbb{I}$ given by

$$
\Delta_{C}^{i_{1}, \ldots, i_{k}}\left(x_{1}, \ldots, x_{n-k+1}\right)=C\left(\mathbf{y}^{\sigma}\right),
$$

where $\mathbf{y}^{\sigma}$ is the vector derived from $\mathbf{y}$ by means of a permutation of its components given by $\sigma:\{1, \ldots, n\} \rightarrow$ $\{1, \ldots, n\}$ where $\sigma(j)=i_{j}$ for $j=1, \ldots, k$, while the other arguments are held fixed. Is it possible to determinate $n$-copulas with given diagonal hyper-plane sections?

\section{Construction of copulas with given opposite diagonal sections}

The opposite diagonal section $\omega_{C}$ of a 2-copula $C$ is a function $\omega_{C}: \mathbb{I} \longrightarrow \mathbb{I}$ defined by $\omega_{C}(t)=C(t, 1-t)$. An opposite diagonal function is a function $\omega: \mathbb{I} \longrightarrow \mathbb{I}$ that satisfies:

(W1) $\omega(t) \leq \min (t, 1-t)$, for all $t \in \mathbb{I}$,

(W2) $\omega$ is 1-Lipschitz, i.e. $\left|\omega(t)-\omega\left(t^{\prime}\right)\right| \leq\left|t-t^{\prime}\right|$ for all $t, t^{\prime}$ in $\mathbb{I}$.

Note that $\omega(0)=\omega(1)=0$. We denote by $\mathcal{O}$ the set of all opposite diagonals.

Observe that there is a bijection between $\mathcal{D}_{2}$ and the set of the opposite diagonals that is given by $\delta \rightarrow \omega_{\delta}$ with $\omega_{\delta}(t):=t-\delta(t)$, so that many of the properties of diagonal functions can be translated to the opposite diagonal functions due to the fact that the copula $C^{\prime}$ defined by $C^{\prime}(u, v):=u-C(u, 1-v)$ for every $(u, v)$ in $\mathbb{I}^{2}$ satisfies $\omega_{C^{\prime}}=\omega_{\delta_{C}}$ (see [20] for more details). Thus, given an opposite diagonal function $\omega$, the existence of a 2-copula $C$ with opposite diagonal section $\omega_{C}$ is guaranteed. It is also possible to define the opposite diagonal splice of two 2-copulas in a similar manner to that in (4).

For a study on the best-possible bounds on sets of copulas with given opposite diagonal sections, see $[58,59]$. 


\section{Construction of copulas with given diagonal and opposite diagonal sections}

In this section we recall necessary and sufficient conditions for a diagonal function $\delta \in \mathcal{D}_{2}$ and an opposite diagonal $\omega \in \mathcal{O}$ be compatible, that is, given $\delta$ and $\omega$, the study of conditions that ensure the existence of a 2-copula $C$ such that $\delta_{C}=\delta$ and $\omega_{C}=\omega$. The first steps in this study were given in [21], where the authors study the compatibility of certain diagonal and opposite diagonal functions with the use of cross-copulas [36] and conjecture the compatibility conditions between $\delta$ and $\omega$.

Let us consider the following assumptions: Let $\delta \in \mathcal{D}_{2}$ and $\omega \in \mathcal{O}$ satisfying:

$$
\left\{\begin{array}{l}
\text { a. } \forall t \in[0,1 / 2], 0 \leq \omega(t)-\delta(t), 0 \leq \omega(1-t)-\delta(t), \\
\text { b. } \forall t \in[1 / 2,1], \delta(t)-\omega(t) \leq 2 t-1, \delta(t)-\omega(1-t) \leq 2 t-1, \\
\text { c. } \forall t, t^{\prime} \in[0,1 / 2], t<t^{\prime} \Rightarrow \delta(t)+\delta(1-t)-\omega(t)-\omega(1-t) \geq \delta\left(t^{\prime}\right)+\delta\left(1-t^{\prime}\right)-\omega\left(t^{\prime}\right)-\omega\left(1-t^{\prime}\right) .
\end{array}\right.
$$

From (7.a) and (7.b), it follows $\delta\left(\frac{1}{2}\right)=\omega\left(\frac{1}{2}\right)$. Taking this equality into account and putting $t^{\prime}=\frac{1}{2}$ in (7.c), we have $\delta(t)+\delta(1-t)-\omega(t)-\omega(1-t) \geq 0$ for all $t \in[0,1 / 2]$.

The solution to the problem comes after finding another equivalent of linear programming, and the tool used is the Farkas' lemma (see, e.g. [81]). So we have

Theorem 9 ([15]). Let $\delta \in \mathcal{D}_{2}$ and $\omega \in \mathcal{O}$ satisfying (7). Then there exists a 2-copula $C$ with diagonal section $\delta$ and opposite diagonal section $\omega$. Moreover, $C$ is unique if, and only if, $\delta(t)=\delta(1-t)-1+2 t=\omega(t)=\omega(1-t)$ for all $t \in[0,1 / 2]$.

Open Problem 5 ([15]). Given a diagonal function and opposite diagonal function that satisfy all the properties that guarantee the existence of at least one copula with these functions as diagonal and opposite diagonal sections, respectively, does there exist a (pointwise) smallest (respectively, greatest) copula with these sections, and if so, can we construct this copula?

\subsection{A generalization: Track and opposite track sections}

The ideas given above in this Section 5 can be applied to problems of compatibility between other types of sections that generalize both the diagonal section and the opposite diagonal section. For this purpose, some preliminary definitions are necessary (for more details, see [43]).

Definition 2. Let $\phi: \mathbb{I} \longrightarrow \mathbb{I}$ be an increasing homeomorphism. We say that the function $\delta_{\phi}: \mathbb{I} \longrightarrow \mathbb{I}$ is a $\phi$-diagonal if it satisfies the following conditions:

(i) $\delta_{\phi}(1)=1$,

(ii) $\delta_{\phi}(t) \leq \min (t, \phi(t))$ for all $t \in \mathbb{I}$,

(iii) $\delta_{\phi}$ is non-decreasing, and

(iv) $\left|\delta_{\phi}\left(t^{\prime}\right)-\delta_{\phi}(t)\right| \leq\left|t^{\prime}-t\right|+\left|\phi\left(t^{\prime}\right)-\phi(t)\right|$ for all $t^{\prime}, t \in \mathbb{I}$.

The graph of the function $\phi$ given in Definition 2, $G_{\phi}$, is called track. Note that if $\phi(t)=t$ for all $t \in \mathbb{I}$, then we have a diagonal function.

Definition 3. Let $\varphi: \mathbb{I} \longrightarrow \mathbb{I}$ be a decreasing homeomorphism. We say that the function $\omega_{\varphi}$ is an opposite $\varphi$-diagonal if $\varphi(1-t)-\omega_{\varphi}(t)$ is a h-diagonal, with $h(t)=\varphi(1-t)$ for all $t \in \mathbb{I}$.

The graph of the function $\varphi$ in Definition 3 is called opposite track. Note that for $h(t)=t$ for all $t \in \mathbb{I}$, the opposite $\varphi$-diagonal is an opposite diagonal. 
The track section $G_{C, \phi}$ of a 2-copula $C$ is the function defined by $G_{C, \phi}(t)=C(t, \phi(t))$ for all $t \in \mathbb{I}$, where $\phi$ is the function given in Definition 2. Similarly, the opposite track section $\omega_{\varphi, C}$ of a copula $C$ is the function defined by $\omega_{\varphi, C}(t)=C(t, \varphi(t))$ for all $t \in \mathbb{I}$, where $\varphi$ is the function given in Definition 3 .

Definition 4. Let $\phi: \mathbb{I} \longrightarrow \mathbb{I}$ be an increasing homeomorphism. The $\phi$-inverse of a set $A \subseteq \mathbb{I}^{2}$ is given by $A^{\phi}:=\left\{(u, v) \in \mathbb{I}^{2}:\left(\phi^{-1}(v), \phi(u)\right) \in A\right\}$.

Note that $A^{\phi}$ is the set of those vertices that constitute the fourth point of a rectangle with sides parallel to the axes, with two opposite vertices on $G_{\phi}$ and a third vertex belonging to $A$.

Definition 5. Let $\phi: \mathbb{I} \longrightarrow \mathbb{I}$ be an increasing homeomorphism, and let $\varphi: \mathbb{I} \longrightarrow \mathbb{I}$ be a decreasing homeomorphism. We say that $\varphi$ is $\phi$-dual if $G_{\varphi}=\left(G_{\varphi}\right)^{\phi}$.

The following theorem generalizes the results in $[15,21]$ by constructing copulas with given track and opposite track sections.

Theorem 10. Let $\phi: \mathbb{I} \longrightarrow \mathbb{I}$ be an increasing homeomorphism, let $\varphi: \mathbb{I} \longrightarrow \mathbb{I}$ be a $\phi$-dual decreasing homeomorphism, let $\delta_{\phi}$ be a $\phi$-diagonal, and let $\omega_{\varphi}$ be an opposite $\varphi$-diagonal. Then there exists a copula $C$ such that $C(t, \phi(t))=\delta_{\phi}(t)$ and $C(t, \varphi(t))=\omega_{\varphi}(t)$ for all $t \in \mathbb{I}$ if, for a given $x_{0} \in \mathbb{I}$ such that $\phi\left(x_{0}\right)=\varphi\left(x_{0}\right)$, we have:

(i) $\omega_{\varphi}(t) \geq \delta_{\phi}(t)$ for all $t \in\left[0, x_{0}\right]$,

(ii) $\omega_{\varphi}\left(\varphi^{-1}(t)\right) \geq \delta_{\phi}\left(\phi^{-1}(t)\right)$ for all $t \in\left[0, \varphi\left(x_{0}\right)\right]$,

(iii) $\delta_{\phi}(t)-\omega_{\varphi}(t) \leq \phi(t)-\varphi(t)$ for all $t \in\left[x_{0}, 1\right]$,

(iv) $\delta_{\phi}\left(\phi^{-1}(t)\right)-\omega_{\varphi}\left(\varphi^{-1}(t)\right) \leq \phi^{-1}(t)-\varphi^{-1}(t)$ for all $t \in\left[\varphi\left(x_{0}\right), 1\right]$,

(v) $\delta_{\phi}\left(\phi^{-1}(\varphi(t))\right)+\delta_{\phi}(t)-\omega_{\varphi}(t)-\omega_{\varphi}\left(\phi^{-1}(\varphi(t))\right)$ is non-increasing in $t \in\left[0, x_{0}\right]$.

Acknowledgement: The authors thank two anonymous referees for valuable comments and acknowledge the support by the Ministry of Economy and Competitiveness (Spain) under research project MTM2014-60594-P (and partially supported by FEDER).

\section{References}

[1] Alsina, C., M. J. Frank, and B. Schweizer (2006). Associative Functions: Triangular Norms and Copulas. World Scientific Publishing, Hackensack NJ.

[2] Alsina, C., R. B. Nelsen, and B. Schweizer (1993). On the characterization of a class of binary operations on distribution functions. Statist. Probab. Lett. 17(2), 85-89.

[3] Arias-García, J. J., B. De Baets, and H. De Meyer (2016). Multivariate Bertino copulas. J. Math. Anal. Appl. 434(2), $1346-1364$.

[4] Arias-García, J. J., R. Mesiar, and B. De Baets (2017). The unwalked path between quasi-copulas and copulas: Stepping stones in higher dimensions. Int. J. Approx. Reason. 80, 89-99.

[5] Arias-García, J. J., R. Mesiar, E. P. Klement, S. Saminger-Platz, and B. De Baets (2018). Extremal Lipschitz continuous aggregation functions with a given diagonal section. Fuzzy Sets Syst., to appear. Available at https://doi.org/10.1016/j.fss.2017.12.014.

[6] Ash, R. B. (2000). Probability and Measure Theory. Second edition. Harcourt/Academic Press, Burlington MA.

[7] Beliakov, G., A. Pradera, and T. Calvo (2007). Aggregation Functions: A Guide for Practitioners. Springer, Berlin.

[8] Bertino, S. (1977). Sulla dissomiglianza tra mutabili cicliche. Metron 35, 53-88.

[9] Butucea, C., J.-F. Delmas, A. Dutfoy, and R. Fischer (2015). Maximum entropy copula with given diagonal section. J. Multivariate Anal. 137, 61-81.

[10] Cherubini, U., E. Luciano, and W. Vecchiato (2004). Copula Methods in Finance. John Wiley \& Sons, Chichester.

[11] Cherubini, U., F. Gobbi, S. Mulinacci, and S. Romagnoli (2012). Dynamic Copula Methods in Finance. John Wiley \& Sons, Chichester.

[12] Cuculescu, I. and R. Theodorescu (2001). Copulas: Diagonals, tracks. Rev. Roumaine Math. Pures Appl. 46(6), $731-742$.

[13] de Amo, E., M. Díaz Carrillo, and J. Fernández-Sánchez (2012). Characterization of all copulas associated with noncontinuous random variables. Fuzzy Sets Syst. 191, 103-112. 
[14] de Amo, E., M. Díaz Carrillo, and J. Fernández-Sánchez (2013). Absolutely continuous copulas with given sub-diagonal section. Fuzzy Sets Syst. 228, 105-113.

[15] de Amo, E., H. De Meyer, M. Díaz Carrillo, and J. Fernández-Sánchez (2016). Characterization of copulas with given diagonal and opposite diagonal sections. Fuzzy Sets Syst. 284, 63-77.

[16] De Baets, B. and H. De Meyer (2007). Orthogonal grid constructions of copulas. IEEE Trans. Fuzzy Syst. 15(6), $1053-1062$.

[17] De Baets, B. and H. De Meyer (2017). A look at copulas in a curved mirror. In M. Úbeda-Flores, E. de Amo Artero, F. Durante, and J. Fernández-Sánchez (Eds.), Copulas and Dependence Models with Applications, pp. 33-47. Springer, Cham.

[18] De Baets, B., H. De Meyer, and R. Mesiar (2007). Asymmetric semilinear copulas. Kybernetika 43(2), 221-233.

[19] De Baets, B., H. De Meyer, and M. Úbeda-Flores (2007). Extremes of the mass distribution associated with a trivariate quasicopula. C. R. Acad. Sci. Paris, Sér. I 344(9), 587-590.

[20] De Baets, B., H. De Meyer, and M. Úbeda-Flores (2009). Opposite diagonal sections of quasi-copulas and copulas. Int. J. Unc. Fuzz. Knowl. Based Syst. 17(4), 481-490.

[21] De Baets, B., H. De Meyer, and M. Úbeda-Flores (2011). Constructing copulas with given diagonal and opposite diagonal sections. Commun. Stat. Theory Methods 40(5), 828-843.

[22] De Luca, G. and G. Rivieccio (2012). Multivariate tail dependence coefficients for Archimedean copulae. In A. Di Ciaccio, M. Coli, and J. M. Angulo Ibañez (Eds.), Advanced Statistical Methods for the Analysis of Large Data-Sets, pp. 287-296. Springer, Berlin.

[23] Denuit, M., J. Dhaene, M. Goovaerts, and R. Kaas (2005). Actuarial Theory for Dependent Risk: Measures, Orders and Models. John Wiley \& Sons, Chichester.

[24] Di Bernardino, E. and D. Rullière (2013). On certain transformations of Archimedean copulas: Application to the nonparametric estimation of their generators. Depend. Model. 1, 1-36.

[25] Durante, F. and J. Fernández-Sánchez (2011a). A note on biconic copulas. Kybernetika 47(4), 532-540.

[26] Durante, F. and J. Fernández-Sánchez (2011b). On the classes of copulas and quasi-copulas with a given diagonal section. Int. J. Unc. Fuzz. Knowl. Based Syst. 19(1), 1-10.

[27] Durante, F. and P. Jaworski (2008). Absolutely continuous copulas with given diagonal sections. Commun. Stat. Theory Methods 37(18), 2924-2942.

[28] Durante, F. and C. Sempi (2016). Principles of Copula Theory. CRC Press, Boca Raton FL.

[29] Durante, F., J. Fernández-Sánchez, and R. Pappadà (2015). Copulas, diagonals, and tail dependence. Fuzzy Sets Syst. 264, 22-41.

[30] Durante, F., J. Fernández-Sánchez, and W. Trutschnig (2014). Multivariate copulas with hairpin support. J. Multivariate Anal. 130, 323-334.

[31] Durante, F., R. Mesiar, and C. Sempi (2006). On a family of copulas constructed from the diagonal section. Soft Comput. 10(6), 490-494.

[32] Durante, F., J. A. Rodríguez-Lallena, and M. Úbeda-Flores (2009). New constructions of diagonal patchwork copulas. Inform. Sci. 179(19), 3383-3391.

[33] Durante, F., S. Saminger-Platz, and P. Sarkoci (2009). Rectangular patchwork for bivariate copulas and tail dependence. Commun. Stat. Theory Methods 38(15), 2515-2527.

[34] Durante, F., J. Fernández-Sánchez, J. J. Quesada-Molina, and M. Úbeda-Flores (2016). Diagonal plane sections of trivariate copulas. Inform. Sci.333, 81-87.

[35] Durante, F., A. Kolesárová, R. Mesiar, and C. Sempi (2007a). Copulas with given diagonal sections: Novel constructions and applications. Int. J. Unc. Fuzz. Knowl. Based Syst. 15(4), 397-410.

[36] Durante, F., A. Kolesárová, R. Mesiar, and C. Sempi (2007b). Copulas with given values on a horizontal and a vertical section. Kybernetika 43(2), 209-220.

[37] Durante, F., A. Kolesárová, R. Mesiar, and C. Sempi (2008). Semilinear copulas. Fuzzy Sets Syst. 159(1), 63-76.

[38] Embrechts, P., A. J. McNeil, and D. Straumann (2002). Correlation and dependence in risk management: Properties and pitfalls. In M. A. H. Dempster (Ed.), Risk Management: Value at Risk and Beyond, pp.176-223. Cambridge University Press.

[39] Erdely, A. and J. M. González-Barrios (2006). On the construction of families of absolutely continuous copulas with given restrictions. Commun. Stat. Theory Methods 35(4), 649-659.

[40] Erdely, A., J. M. González-Barrios, and M. M. Hernández-Cedillo (2014). Frank’s condition for multivariate Archimedean copulas. Fuzzy Sets Syst. 240, 131-136.

[41] Fernández-Sánchez, J. and W. Trutschnig (2016). Some members of the class of (quasi-)copulas with given diagonal from the Markov kernel perspective. Commun. Stat. Theory Methods 45(5), 1508-1526.

[42] Fernández-Sánchez, J. and M. Úbeda-Flores (2016). The distribution of the probability mass of biconic copulas. Inform. Sci. 329, 177-183.

[43] Fernández-Sánchez, J. and M. Úbeda-Flores (2017). Copulas with given track and opposite track sections: Solution to a problem on diagonals. Fuzzy Sets Syst. 308, 133-137.

[44] Fernández-Sánchez, J., R. B. Nelsen, and M. Úbeda-Flores (2011). Multivariate copulas, quasi-copulas and lattices. Statist. Probab. Lett. 81(9), 1365-1369.

[45] Fernández-Sánchez, J., J. A. Rodríguez-Lallena, and M. Úbeda-Flores (2011). Bivariate quasi-copulas and doubly stochastic signed measures. Fuzzy Sets Syst. 168(1), 81-88. 
[46] Fernández-Sánchez, J., R. B. Nelsen, J. J. Quesada-Molina, and M. Úbeda-Flores (2016). Independence results for multivariate tail dependence coefficients. Fuzzy Sets Syst. 284, 129-137.

[47] Frank, M. J. (1996). Diagonals of copulas and Schröder's equation. Aequationes Math. 51(1-2), 150.

[48] Fredricks, G. A. and R. B. Nelsen (1997). Copulas constructed from diagonal sections. In V. Beneš and J. Štěpán (Eds.), Distributions with Given Marginals and Moment Problems, pp. 129-136. Springer, Dordrecht.

[49] Fredricks, G. A. and R. B. Nelsen (2002). The Bertino family of copulas. In C. M. Cuadras, J. Fortiana, and J. A. RodríguezLallena (Eds.), Distributions with Given Marginals and Statistical Modelling, pp. 81-91. Springer, Dordrecht.

[50] Genest, C., J. J. Quesada-Molina, J. A. Rodríguez-Lallena, and C. Sempi (1999). A characterization of quasi-copulas. J. Multivariate Anal. 69(2), 193-205.

[51] Grabisch, M., J.-L. Marichal, R. Mesiar, and E. Pap (2009). Aggregation Functions. Cambridge University Press.

[52] Jakob, K. and M. Fischer (2014). Quantifying the impact of different copulas in a generalized CreditRisk ${ }^{+}$framework: An empirical study. Depend. Model. 2, 1-21.

[53] Jaworski, P. (2009). On copulas and their diagonals. Inform. Sci. 179(17), 2863-2871.

[54] Jaworski, P. and T. Rychlik (2008). On distributions of order statistics for absolutely continuous copulas with applications to reliability. Kybernetika 44(6), 757-776.

[55] Joe, H. (1997). Multivariate Models and Dependence Concepts. Chapman \& Hall, London.

[56] Jwaid, T., B. De Baets, and H. De Meyer (2009). Orbital semilinear copulas. Kybernetika 45(6), 1012-1029.

[57] Jwaid, T., B. De Baets, and H. De Meyer (2012). Biconic aggregation functions. Inform. Sci. 187, 129-150.

[58] Klement, E. P. and A. Kolesárová (2005a). 1-Lipschitz aggregation operators, quasi-copulas and copulas with given opposite diagonal. In B. Reusch (Ed.), Computational Intelligence, Theory and Applications, pp. 565-572. Springer, Berlin.

[59] Klement, E. P. and A. Kolesárová (2005b). Extension to copulas and quasi-copulas as special 1-Lipschitz aggregation operators. Kybernetika 41(3), 329-348.

[60] Klement, E. P. and A. Kolesárová (2007). Intervals of 1-Lipschitz aggregation operators, quasi-copulas, and copulas with given affine section. Monatsh. Math. 152(2), 151-167.

[61] Li, H. (2009). Orthant tail dependence of multivariate extreme value distributions. J. Multivariate Anal.100(1), 243-256.

[62] Malevergne, Y. and D. Sornette (2006). Extreme Financial Risks. From Dependence to Risk Management. Springer, Berlin.

[63] Mayor, G. and J. Torrens (1991). On a family of $t$-norms. Fuzzy Sets Syst. 41(2), 161-166.

[64] Mayor, G., R. Mesiar, and J. Torrens (2008). On quasi-homogeneous copulas. Kybernetika 44(6), 745-756.

[65] McNeil, A. J. and J. Nešlehová (2009). Multivariate Archimedean copulas, $d$-monotone functions and $l_{1}$-norm symmetric distributions. Ann. Statist. 37(5B), 3059-3097.

[66] McNeil, A. J., R. Frey, and P. Embrechts (2005). Quantitative Risk Management: Concepts, Techniques and Tools. Princeton University Press.

[67] Mesiar, R. and J. Kalická (2013). Diagonal copulas. In H. Bustince, J. Fernández, R. Mesiar, and T. Calvo (Eds.), Aggregation Functions in Theory and in Practise, pp. 67-74. Springer, Heidelberg.

[68] Mesiar, R. and M. Navara (1999). Diagonals of continuous triangular norms. Fuzzy Sets Syst. 104(1), 35-41.

[69] Mesiar, R. and C. Sempi (2010). Ordinal sums and idempotents of copulas. Aequationes Math. 79(1-2), 39-52.

[70] Nelsen, R. B. (2005). Copulas and quasi-copulas: An introduction to their properties and applications. In E. P. Klement and R. Mesiar (Eds.), Logical, Algebraic, Analytic, and Probabilistic Aspects of Triangular Norms, pp. 391-413. Elsevier, Amsterdam.

[71] Nelsen, R. B. (2006). An Introduction to Copulas. Second edition. Springer, New York.

[72] Nelsen, R. B. and G. A. Fredricks (1997). Diagonal copulas. In V. Beneš and J. Štěpán (Eds.), Distributions with Given Marginals and Moment Problems, pp. 121-128. Springer, Dordrecht.

[73] Nelsen, R. B. and M. Úbeda-Flores (2005). The lattice-theoretic structure of sets of bivariate copulas and quasi-copulas. $C$. R. Acad. Sci. Paris, Sér. I 341(9), 583-586.

[74] Nelsen, R. B., J. J. Quesada-Molina, J. A. Rodríguez-Lallena, and M. Úbeda-Flores (2002). Some new properties of quasicopulas. In C. M. Cuadras, J. Fortiana, and J. A. Rodríguez-Lallena (Eds.), Distributions with Given Marginals and Statistical Modelling, pp. 187-194. Springer, Dordrecht.

[75] Nelsen, R. B., J. J. Quesada-Molina, J. A. Rodríguez-Lallena, and M. Úbeda-Flores (2004). Best-possible bounds on sets of bivariate distribution functions. J. Multivariate Anal. 90(2), 348-358.

[76] Nelsen, R. B., J. J. Quesada-Molina, J. A. Rodríguez-Lallena, and M. Úbeda-Flores (2008). On the construction of copulas and quasi-copulas with given diagonal sections. Insurance Math. Econom. 42(2), 473-483.

[77] Nelsen, R. B., J. J. Quesada-Molina, J. A. Rodríguez-Lallena, and M. Úbeda-Flores (2010). Quasi-copulas and signed measures. Fuzzy Sets Syst. 161(17), 2328-2336.

[78] Nelsen, R. B., J. J. Quesada-Molina, B. Schweizer, and C. Sempi (1996). Derivability of some operations on distribution functions. In L. Rüschendorf, B. Schweizer, and M. D. Taylor (Eds.), Distributions with Fixed Marginals and Related Topics, pp. 233-243. Institute of Mathematical Statistics, Hayward CA.

[79] Quesada-Molina, J. J., S. Saminger-Platz, and C. Sempi (2008). Quasi-copulas with a given sub-diagonal section. Nonlinear Anal. 69(12), 4654-4673.

[80] Rodríguez-Lallena, J. A. and M. Úbeda-Flores (2004). Best-possible bounds on sets of multivariate distribution functions. Commun. Stat. Theory Methods 33(4), 805-820.

[81] Schrijver, A. (1998). Theory of Linear and Integer Programming. John Wiley \& Sons, Chichester. 
[82] Seethoff, T. L. and R. C. Shiflett (1978). Doubly stochastic measures with prescribed support. Z. Wahrscheinlichkeitstheorie Verw. Gebiete 41(4), 283-288.

[83] Segers, J. (2011). Comments on: Inference in multivariate Archimedean copula models. Test 20, 281-283.

[84] Sempi, C. (2017). Quasi-copulas: a brief survey. In M. Úbeda-Flores, E. de Amo Artero, F. Durante, and J. Fernández-Sánchez (Eds.), Copulas and Dependence Models with Applications, pp. 203-224. Springer, Cham.

[85] Shannon, C. E. (1948). A mathematical theory of communication. Bell Syst. Tech. J. 27, 379-423.

[86] Sherwood, H. and M. D. Taylor (1988). Doubly stochastic measures with hairpin support. Probab. Theory Related Fields 78 , 617-626.

[87] Sklar, A. (1959). Fonctions de répartition à $n$ dimensions et leurs marges. Publ. Inst. Statist. Univ. Paris 8, 229-231.

[88] Stupňanová, A. and A. Kolesárová (2011). Associative $n$-dimensional copulas. Kybernetika 47(1), 93-99.

[89] Sungur, E. A. and Y. Yang (1996). Diagonal copulas of Archimedean class. Commun. Stat. Theory Methods 25(7), $1659-1676$.

[90] Tankov, P. (2011). Improved Fréchet bounds and model-free pricing of multi-asset options. J. Appl. Probab. 48(2), 389-403.

[91] Úbeda-Flores, M. (2001). Cópulas y Cuasicópulas: Interrelaciones y Nuevas Propiedades. Aplicaciones. Ph.D. thesis, Universidad de Almería.

[92] Úbeda-Flores, M. (2008). On the best-possible upper bound on sets of copulas with given diagonal sections. Soft Comput. 12(10), 1019-1025.

[93] Úbeda-Flores, M. and J. Fernández-Sánchez (2017). Sklar’s theorem: The cornerstone of the Theory of Copulas. In M. ÚbedaFlores, E. de Amo Artero, F. Durante, and J. Fernández-Sánchez (Eds.), Copulas and Dependence Models with Applications, pp. 241-258. Springer, Cham.

[94] Wysocki, W. (2012). Constructing Archimedean copulas from diagonal sections. Statist. Probab. Lett. 82(4), 818-826.

[95] Wysocki, W. (2015). Characterizations of Archimedean n-copulas. Kybernetika 51(2), 212-230. 